\section{Internationale Abfallwirtschaft}

Diskutiert man Kreisläufe beim Recycling von Abfällen, so versteht man darunter in der Regel materielle Kreisläufe: Aus einem Rohstoff entstehen Produkte, die konsumiert werden und entweder als Güter wiederverwendet oder als Stoffe verwertet werden können. Stoffkreisläufe haben aber auch eine geografische Komponente: Rohstoffe werden beispielsweise in Afrika gewonnen, in Europa oder den USA genutzt, und gelangen als Abfälle, z. B. Elektronikschrott, wieder nach Afrika zurück. Solche räumlich definierte Kreisläufe sind wirtschaftlich sinnvoll, da sie die Standortvorteile der unterschiedlichen Akteure berücksichtigen: Die Produzentenländer verfügen über eine solide Rohstoffbasis und können dank relativ geringer Gewinnungskosten ihre Produkte zu günstigen Preisen auf dem Weltmarkt anbieten. Die Kosten des Recyclings gewisser Abfälle in hochentwickelten Ländern des Nordens sind derzeit zu hoch, als dass diese sich stark beim Recycling engagieren. Für mehrere Abfallarten ist es wirtschaftlicher, sie in Drittweltländern manuell aufzuarbeiten, und wertvolle Sekundärrohstoffe daraus zu gewinnen. Dies wird sich in wenigen Jahren ändern, wenn in Europa die groBen, stark wachsenden Lager an Konsumprodukten den Abfallmarkt überschwemmen werden und Hightech-Recyclingprozesse auf breiter Basis verfügbar werden.

Kreisläufe dürfen nicht nur unter wirtschaftlichen und geografischen Gesichtspunkten betrachtet werden. Ebenso wichtig sind ökologische Aspekte: Durch die Gestaltung von Stoffkreisläufen können in aller Regel große Mengen an Energie, Rohstoffen, $\mathrm{CO}_{2}$-Emissionen und ganz allgemein an „Naturverbrauch" eingespart werden. Dies gilt grundsätzlich auch für ein Recycling in Afrika oder anderen weniger entwickelten Kontinenten. Allerdings sieht die Praxis derzeit oft anders aus: Der Stand der Technik wird nur selten erreicht, die Arbeitsbedingungen sind schlecht, eine Gesundheitsvorsorge fehlt oft, und die resultierende Belastung von Mensch und Umwelt ist unakzeptabel. Für Unternehmungen des Nordens, die sich europäischen Umwelt- und Gesundheitsstandards unterziehen, erwächst ein eklatanter Wettbewerbsnachteil. Die Basler Konvention versucht Abhilfe zu schaffen, allerdings ist in der Praxis der Erfolg noch nicht flächendeckend.

Es liegt im Interesse aller in der Abfallwirtschaft beteiligten Akteure, Kreisläufe auf ein solides, ausgewogenes Fundament zu stellen. Grundlage dazu ist eine zielorientierte Wissensbasis, die die wesentlichsten Bereiche umfasst. „Zielorientiert“ bedeutet, dass Informationen nicht wahllos, sondern fokussiert auf die Ziele der Abfallwirtschaft, d. h. den Schutz von Mensch und Umwelt sowie die optimale Ressourcennutzung, gesammelt werden. Solche Wissensbasen bestehen in hochentwickelten Ländern in vielen Bereichen, fehlen aber größtenteils in Entwicklungsländern. Besonders bedauerlich ist, dass das methodische Know-how fehlt, wie das notwendige Wissen erarbeitet werden kann. Die Zusammenarbeit mit den Entwicklungsländern ist in der heutigen Situation deshalb vor allem auch für Hochschulen und tertiäre Bildungsinstitutionen aus dem Norden, die über das notwendige methodische Rüstzeug verfügen, wichtig.

Notwendiges Wissen umfasst makro- und mikroökonomische Daten über Volkseinkommen und abfallwirtschaftliche Prozesse, Angaben über Abfälle, deren Entstehung und Behandlungsmöglichkeiten, über regionale Gegebenheiten wie auch über die Interessen der verschiedenen Akteursgruppen aus Bevölkerung, Politik, Unternehmungen und Verwaltung. Dieses Wissen stammt aus verschie-

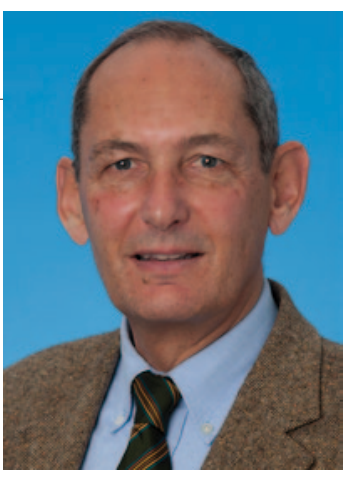

o.Univ.-Prof. DI Dr. Paul H. Brunner 
denen Disziplinen, es zu erarbeiten ist eine anspruchsvolle inter- und transdisziplinäre Aufgabe. Die meisten Länder, die noch über keine abfallwirtschaftliche Wissensbasis verfügen, weisen nur ein geringes volkswirtschaftliches Potenzial auf. Es ist deshalb viel Überzeugungsarbeit zu leisten, damit sie die geringen Mittel, die innen für die Abfallwirtschaft zur Verfügung stehen - man rechnet mit rund $0,3 \%$ des Bruttosozialproduktes - in die prioritäre Erstellung einer einfachen Ansprüchen genügenden Wissensbasis investieren. Damit denke ich nicht an eine der häufig anzutreffenden Planungsstudien, die von hochentwickelten Gesellschaften und ihrer Denk- und Handlungsweise ausgehen, sondern an transdisziplinäre Prozesse zur Gewinnung von lokalen Informationen, die neben Wissen auch Bewusstsein für abfallwirtschaftliche Probleme erzeugen.

In der vorliegenden Ausgabe der „Österreichischen Wasser- und Abfallwirtschaft" tragen fünf Autorenteams zur Darstellung und Lösung der Problematik der Abfallwirtschaft in Entwicklungsländern bei. Notgedrungen müssen dies einzelne Mosaiksteine bleiben, die Thematik ist viel zu groß und zu komplex. Allerdings zeigen die Beiträge, dass mit seriöser Forschungsarbeit wesentliche Fragen herausgearbeitet werden können, und auch Antworten für zukünftige Lösungen resultieren. Da die Ziele der europäischen Abfallwirtschaft bereits größtenteils erreicht sind, sollte sich unser Interesse vermehrt den Ländern im Süden, in denen noch ein sehr großer abfallwirtschaftlicher Nachholbedarf besteht, zuwenden.

o.Univ.-Prof. DI Dr. Paul H. Brunner

Technische Universität Wien

Institut für Wassergüte, Ressourcenmanagement und Abfallwirtschaft

Karlsplatz 13/226

1040 Wien

Österreich

paul.h.brunner@tuwien.ac.at 